\title{
One Genetic Defect and Two Related Entities in Monozygotic Twins: Otosclerosis and Superior Semicircular Canal Near Dehiscence Syndrome
}

\author{
F Ceyda Akin Ocal ${ }^{1}$, Haluk Kavus ${ }^{2}$, Bulent Satar ${ }^{3}$, and Davut Pehlivan ${ }^{4,5}$ \\ Departments of ${ }^{1}$ Otorhinolaryngology and ${ }^{2}$ Medical Genetics, University of Health Sciences, Gulhane Training and Research Hospital, \\ Ankara, Turkey \\ ${ }^{3}$ Department of Otorhinolaryngology, University of Health Sciences, Gulhane Medical School, Ankara, Turkey \\ ${ }^{4}$ Department of Pediatrics, Section of Pediatric Neurology and Developmental Neuroscience, Baylor College of Medicine, \\ Houston, TX, USA \\ ${ }^{5}$ Department of Molecular and Human Genetics, Baylor College of Medicine, Houston, TX, USA
}

\author{
Received June 18, 2021 \\ Revised August 17, 2021 \\ Accepted September 4, 2021
}

Address for correspondence

F Ceyda Akin Ocal, MD

Department of Otorhinolaryngology,

University of Health Sciences,

Gulhane Traning and

Research Hospital,

Ankara 38000,Turkey

Tel +90 5058227471

E-mail fceydaakin@gmail.com
The purpose of this study was to evaluate the clinical and genetic findings of 53-year-old monozygotic twins who had bilateral otosclerosis and right-sided superior semicircular canal near dehiscence (SSCND). Monozygotic twins at the age of 53 presented with conductive hearing loss and normal tympanic membranes. Detailed audiovestibular testing and computed tomography scan revealed that both patients had concurrent otosclerosis and SSCND. Conservative management (hearing aids) was the treatment for these patients. Exome sequencing (ES) for the twins and their affected mother identified a heterozygous missense variant in the EYA4 (c.1744G>A; p.Glu582Lys) gene. This is the first case report to present these separate entities identified in monozygotic twins with a heterozygous missense variant in the EYA4 gene. Our ES data may imply a possible causal relationship or association between variants in the EYA4 gene and concurrent otosclerosis and SSCND. J Audiol Otol 2022;26(2):97-102

Keywords: Otosclerosis; Superior semicircular canal dehiscence syndrome; Hearing loss; Genetic analysis; Exome sequencing.

\section{Introduction}

Patients with concomitant otosclerosis and superior semicircular canal dehiscence (SSCD) have been rarely described in the literature. There is no known genetic or biological link between these two disorders. When the causes of failure in hearing gain after stapes surgery in patients with otosclerosis were first investigated, the presence of accompanying SSCD was revealed in some cases [1]. In cases where otosclerosis and SSCD occur concurrently, clinical findings resemble those found in otosclerosis (normal otoscopy, progressive conductive hearing loss [CHL], and absence of acoustic reflex). More importantly, there was no evidence of the third window (bone-

This is an Open Access article distributed under the terms of the Creative Commons Attribution Non-Commercial License (https://creativecommons.org/licenses/by-nc/4.0/) which permits unrestricted non-commercial use, distribution, and reproduction in any medium, provided the original work is properly cited. conduction hyperacusis, autophony, or sound- or pressure-induced vertigo). The reason behind this is that one of the three windows in SSCD is closed due to otosclerosis. Also in the literature patients with superior semicircular canal near dehiscence (SSCND) had similar symptoms with frank dehiscence [2].

This case report aims to propose a genetic defect for both otosclerosis and SSCND in the light of the molecular analysis of affected monozygotic twins. The coexistence of otosclerosis and SSCND has not been shown to have a genetic etiology so far. We saw both phenotypes in monozygotic twins and their affected mother and were able to identify a possible genetic variant that may have contribute the development of these diseases.

\section{Case Report}

Two monozygotic twin male patients, at the age of 53, were 
Two Related Entities in Monozygotic Twins: Otosclerosis and Superior Semicircular Canal Near Dehiscence Syndrome

admitted to our inpatient service with a long-standing and gradually increasing hearing loss complaint. Both patients had bilateral progressive hearing loss and bilateral pulsatile tinnitus. Twin 1 had autophonia but was not present in the twin 2 .
Both of them had hyperacusis. Neither patients had vertigo nor any history of trauma. Their mother had a very similar course but she was not available for clinical evaluation.

Otoscopic examinations of both patients were completely
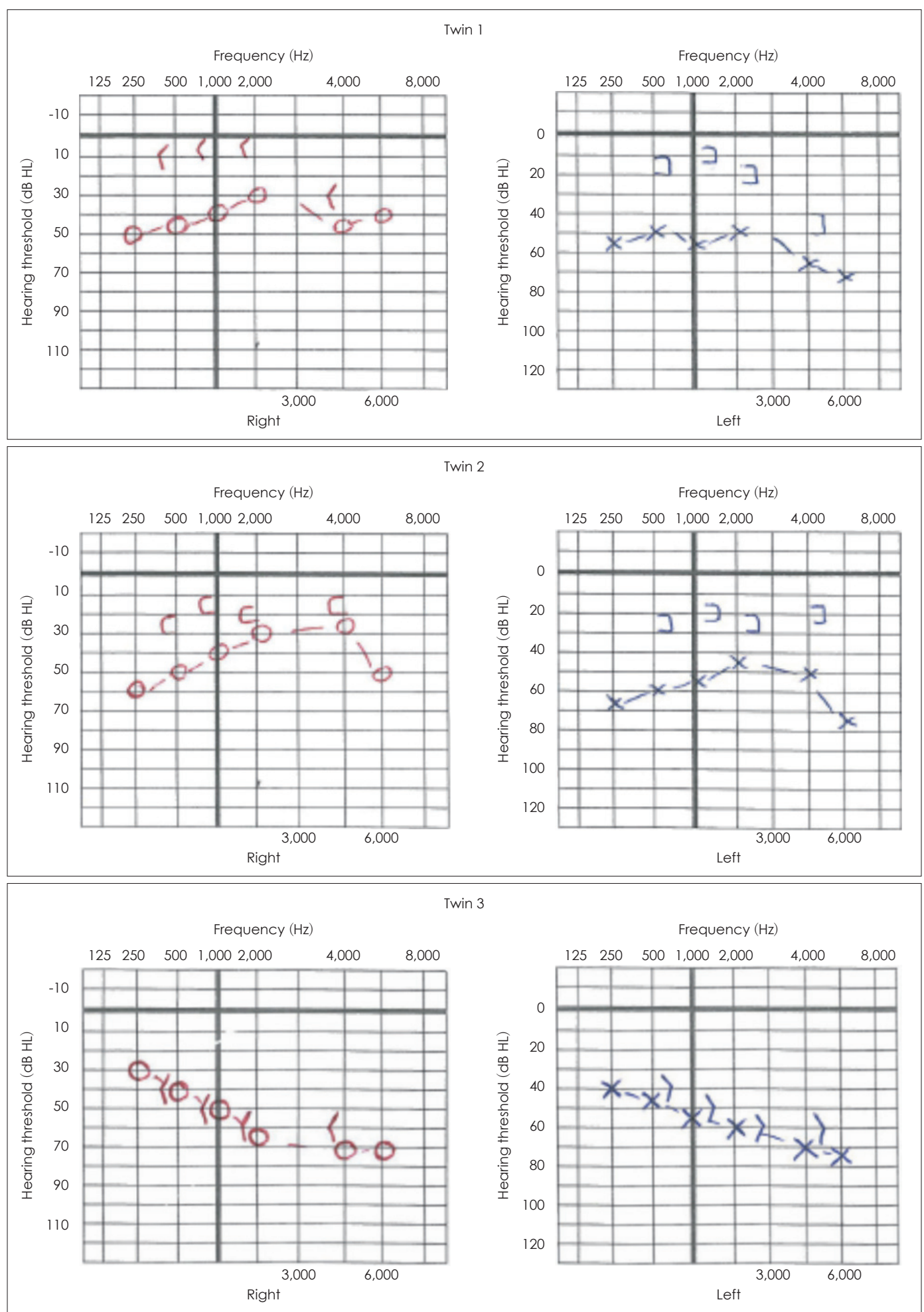

Fig. 1. The audiograms of the twins and their mother's. 
normal, and their audiograms showed mild to moderate CHL greater for low frequencies in both sides (Fig. 1). Table 1 summarizes the diagnostic studies.

The computed tomography (CT) scan of the twins revealed bilateral otosclerosis and right-sided SSCND (Fig. 2). Diagnosis of otosclerosis is based on CHL, absence of acoustic reflex, unresponsiveness to air-conduction cervical vestibular evoked myogenic potential (c-VEMP), and fenestral otosclerosis in one of the patients and suspected otosclerosis in another one in the CT scan.

Middle ear exploration and stapes surgery, when necessary, and hearing aid were offered as alternatives. In addition, the patients were informed of the fact that SSCND findings might become evident after stapedotomy. Because the operation may uncover a third window. Both patients preferred amplification.

Exome sequencing (ES) was performed on all three affected individuals (twins and mother) (Fig. 3) using standard sequencing and variant prioritization workflow that has been previously published (Supplementary Table 1 in the onlineonly Data Supplement). We identified a heterozygous missense variant (NM_172105.3; c.1744G $>$ A [p.Glu582Lys]) in the eyes absent 4 (EYA4) gene (Fig. 3A). The affected glutamic acid residue is within the Eya-homologous region (EyaHR) domain and highly-conserved throughout species (Fig. 3B). The variant is absent from the public variant databases, including gnomAD, ExAC, ARIC, and ESP [3-6]. Bioinformatic analyses were used to predict potential variant effects on protein function (SIFT, PolyPhen2, MutationTaster, Phylop, CADD). Validation and segregation studies are performed using Sanger sequencing (Fig. 3A).

This study has been conducted in accordance with the Helsinki Declaration of Principles. Written informed consent forms were obtained from the twins and their mothers.

\section{Discussion}

Although the coexistence of otosclerosis and SSCD or SSCND findings is rarely seen in the literature, it has been reported that the underlying pathogeneses are different and it is coincidental that they are found simultaneously in the same patient $[7,8]$. The prevalence of concomitant otosclerosis and SSCD findings has been reported to be 6 to 8 per 100,000 [8]. It is known that there may be congenital or acquired causes in the development of SSCD, but congenital causes are prominent, and familial cases of SSCD syndrome have been reported [9]. It has been shown that SSCND may have the same effect as the frank dehiscence observed. Environmental and patient-specific factors such as estrogen exposure and viral infection play a minor role in the pathogenesis of otosclerosis, but genetic factors likely play the biggest role [10]. Otosclerosis is considered a complex disease with rare autosomal dominant forms caused by a single gene. TGF-B1 pathway is probably an important factor in the pathogenesis of otosclerosis [10]. Therefore, identifying these diseases together in mono-

Table 1. Diagnostic studies of the patients

\begin{tabular}{|c|c|c|}
\hline & Twin 1 & Twin 2 \\
\hline Weber & Lateralized to the left & Midline \\
\hline Rinne $(512 \mathrm{~Hz})$ & Bilaterally negative & Bilaterally negative \\
\hline Tullio phenomenon & - & - \\
\hline Hennebert's sign & - & - \\
\hline $\begin{array}{l}\text { Averaged air conducted and bone conducted } \\
\text { thresholds }(0.5,1,2 \text { and } 4 \mathrm{kHz})\end{array}$ & R: $38 / 7 \mathrm{~dB}, \mathrm{~L}: 52 / 15 \mathrm{~dB}$ & R: $40 / 20 \mathrm{~dB}, \mathrm{~L}: 53 / 23 \mathrm{~dB}$ \\
\hline $\begin{array}{l}\text { Speech reception threshold/word recognition } \\
\text { score }\end{array}$ & R: 35 dB, L: 50 dB/R: 96\%, L: 96\% & R: 40 dB, L: 55 dB/R: 88\%, L: $88 \%$ \\
\hline Acoustic reflex & Bilaterally absent & Bilaterally absent \\
\hline Timpanogram & Type A & Type A \\
\hline $\begin{array}{l}\text { VNG (saccade, smooth pursuit, optokinetic, } \\
\text { gaze positional, spontaneous nystagmus, etc) } \\
\text { and caloric test }\end{array}$ & Normal & Normal \\
\hline Air-conduction c-VEMP (500 Hz tone burst) & Bilaterally absent & Bilaterally absent \\
\hline Bone-conduction oVEMP (500 Hz tone burst) & $\begin{array}{l}\text { Higher amplitude and lower threshold } \\
\text { on the right side ( } 50 \mathrm{~dB} \mathrm{nHL} \text { ), } \\
\text { no response on the left side }\end{array}$ & $\begin{array}{l}\text { Higher amplitude and lower threshold } \\
\text { on the right side ( } 50 \mathrm{~dB} \mathrm{nHL} \text {, } \\
\text { no response on the left side }\end{array}$ \\
\hline Transient otoacoustic emission & No response & No response \\
\hline
\end{tabular}



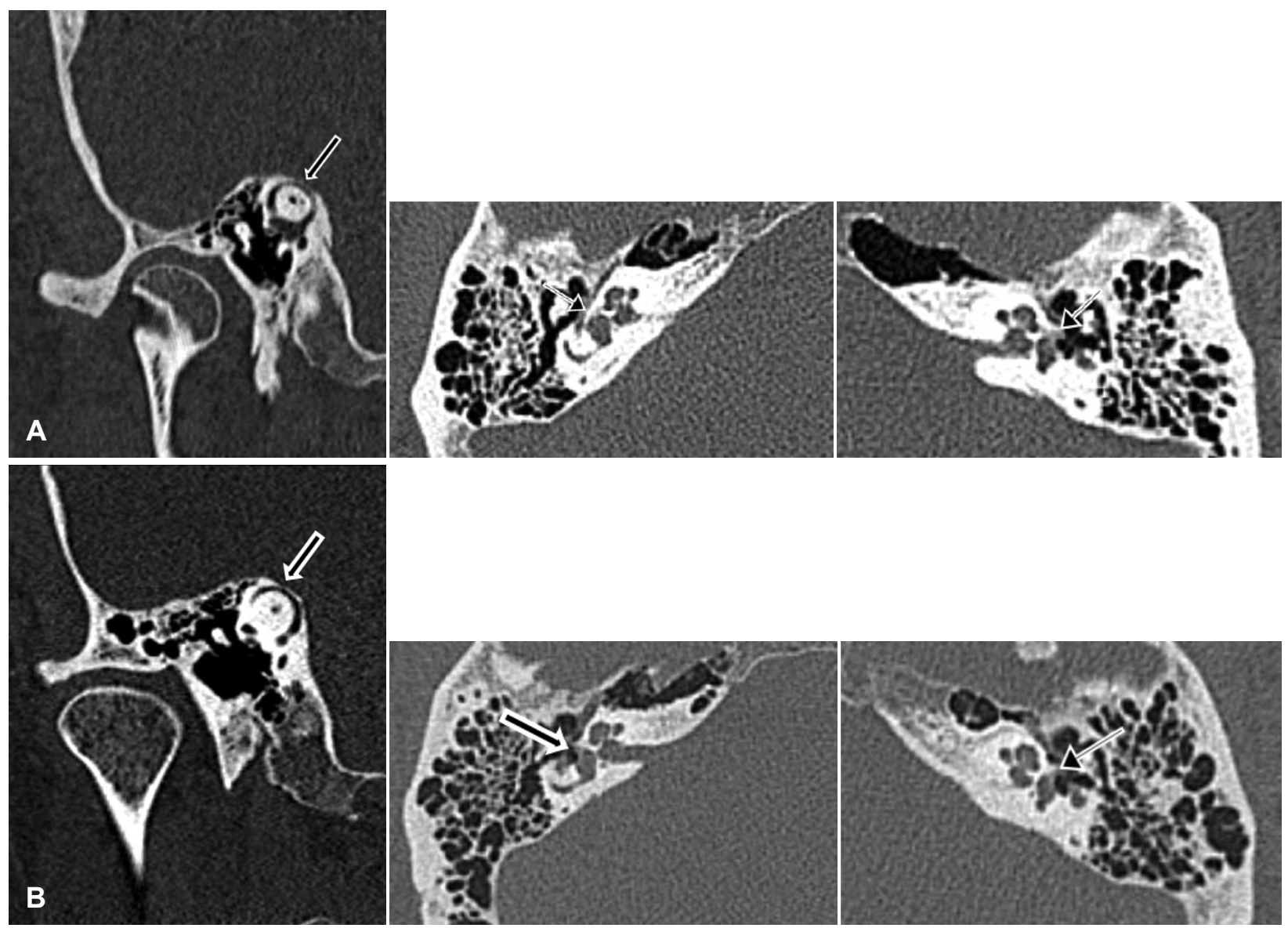

Fig. 2. Temporal bone CT images for the twins. A: Twin 1. White arrows point out the near dehiscence of the superior semicircular canal on the right side (Stenvers view) and possible foci of fenestral otosclerosis on the right and left sides (axial views). B: Twin 2. White arrows point out the near dehiscence of the superior semicircular canal on the right side (Stenvers view) and possible foci of fenestral otosclerosis on the right and left sides (axial views).

zygotic twins, as in this case-report, may be important for the literature.

The coexistence of SSCND and otosclerosis makes it difficult to interpret the clinical and audiological findings. Hope and Fagan [7] reported that SSCD had been detected after sequential stapes surgeries in CT and VEMP examinations of the patient whose air-bone gap improved to $10 \mathrm{~dB}$ as opposed to his mildly affected balance and tinnitus. The simultaneous occurrence of otosclerosis and SSCD syndrome can mask the findings of SSCD syndrome. This was explained by the fact that the three windows, which normally occur with the presence of SSCD syndrome, fall into two windows due to the presence of otosclerosis. Therefore, Hennebert's sign and Tullio phenomenon, acoustic reflex, and air-conduction (AC) VEMP findings observed in SSCD syndrome are not seen in these patients. Auditory symptoms were prominent and vestibular symptoms were less severe in our patients. Hennebert's sign and Tulio phenomenon, acoustic reflex, and AC VEMP findings were not observed. However, high amplitude and low threshold in bone-conduction ocular vestibular evoked myogenic potential (BC-oVEMP) were found to be compatible with SSCD. Thus, the twins had radiographic SSCND with vestibular testing supporting as indicated by higher thresholds on BC-oVEMP testing. They presented with symptoms of otosclerosis. If $\mathrm{CT}$ is not performed routinely in patients with CHL, SSCD or SSCND go unnoticed, which leads the patients to be diagnosed with otosclerosis only. Therefore, we obtain $\mathrm{CT}$ for all patients who have CHL or been diagnosed with otosclerosis based on clinical history and audiological findings in our department. But it should be noted that the quality of CT scan, variability of imaging protocols and interpretations lead to limitations in the SSCD diagnosis. Therefore it should be evaluated together with specific symptoms to the disease or VEMP tests.

One may question the certainty of diagnosis of otosclerosis based on clinical and audiologic findings only instead of intraoperative confirmation in our cases. In the literature, diagnosis of otosclerosis is usually made clinically according to the 

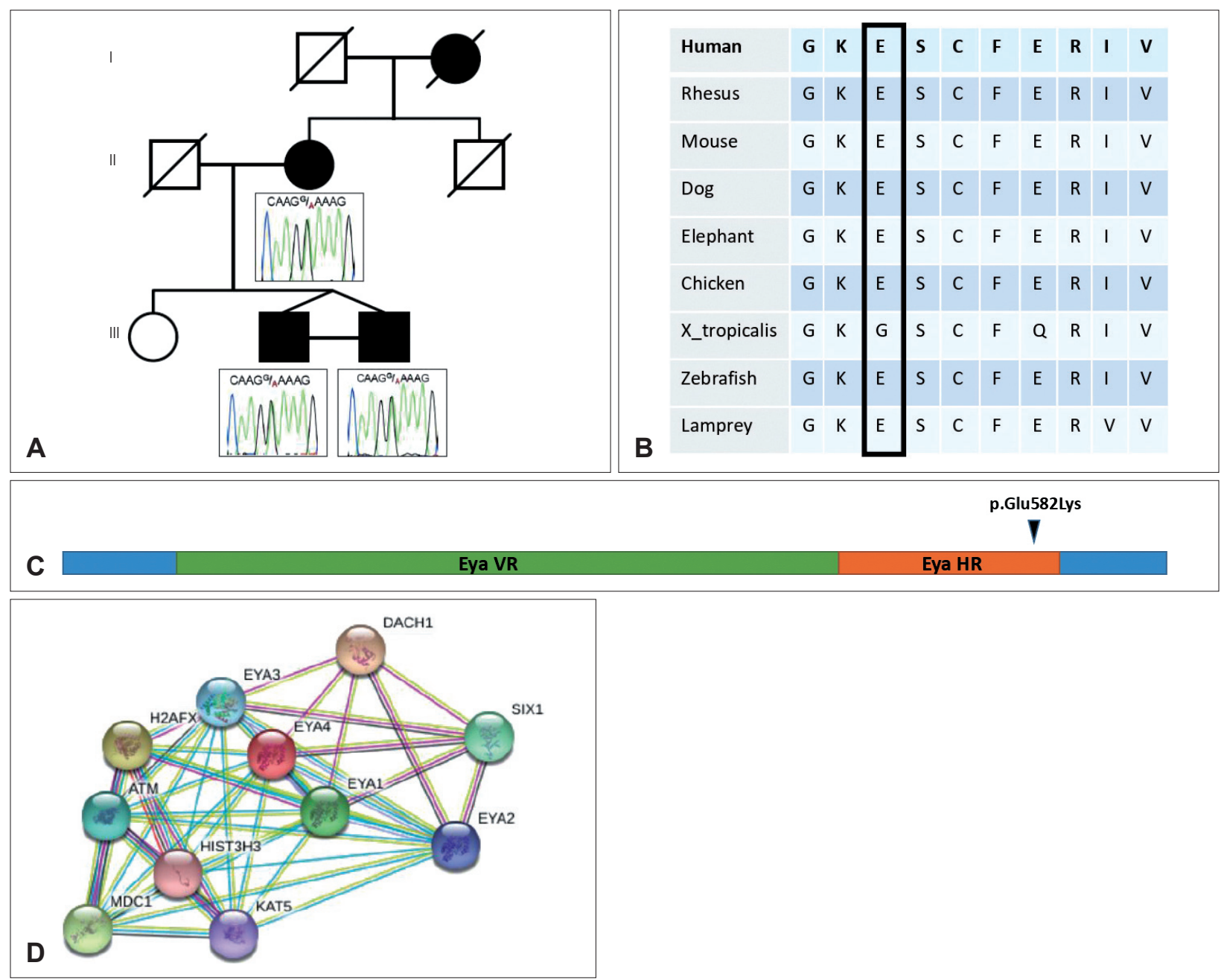

Fig. 3. Molecular genetic studies in the family. A: Sanger validation and segregation of the identified variant monoallelic inherited heterozygous EYA4 (NM_172105.3); c.1744G>A; p.E582K (6-133846158-G-A). B: Evolutionary conservation of the altered amino acid residue at position $58 \overline{2}(E)$. Our position is highlighted with a vertical rectangle. Each letter denotes a specific amino acid. Species throughout the evolution are on the left column. C: Depiction of the domains of EYA4 protein. Each domain is represented with a different color horizontal bar. The position of our variant is shown with an arrow. D: Interacting proteins for EYA4 (STRING Interaction Network Preview). Note the very close interaction between EYA1 and EYA4. Eya-VR, Eya-variable region; Eya-HR, Eya-homologous region.

history of the patient, physical examination, and audiometric evaluation (conductive or mixed type hearing loss, absence of acoustic reflex, and normal otoscopy). Radiological evaluation is not required for the diagnosis, but it confirms the diagnosis of otosclerosis. It is particularly useful in determining SSCD or SSCND in a patient with third window symptoms. However, a normal CT scan does not exclude otosclerosis [11].

In a case report published in 2012, otosclerotic foci of various sizes were found in the postmortem temporal bone. One of the foci was seen in the superior semicircular canal (SSC) [12].

Our case also suggests that site of near dehiscense in the SSC may be another otosclerotic focus.

Family-based ES revealed a heterozygous missense variant in the EYA4 gene. Variants in the EYA4 gene cause deafness, autosomal dominant 10 (DFNA10) (MIM: \#601316). EYA4 encodes a 639-amino acid protein containing a highly conserved C-terminal domain of 271 amino acids, which has been designated the Eya-HR domain (Fig. 3C). In Drosophila, eya is known to mediate developmentally important protein-protein interactions. In the developing mouse embryo, Eya4 was expressed primarily in the craniofacial mesenchyme, the dermamyotome, and the limb [13]. Another member of the EYA family, EYA1, a paralog and close interactor of EYA4, has a role in the development of ear structures, and mutations in this gene cause Branchiootic syndrome 1 (MIM \#602588) (Fig. $3 \mathrm{D})$ as well as other disorders causing craniofacial anomalies. Depreux, et al. [14] showed that Eya4 expressed in mouse developing SSCs and cochlea along with a strong expression in the middle ear-forming region and surrounding the first branchial pouch, from which eustachian tube structures were 
derived. Also Gana, et al. [15] reported that EYA4 is a good candidate for otofaciocervical syndrome (OTFCS) according to its pattern of expression, its sequence similarity to EYA1, and its involvement in Pax-Six-Eya-Dach network (PSEDN). Given the EYA4 gene's role in the development of SSC and cochlea (mouse data), and dysfunction of close interacting gene's, EYA1, causing otofacial anomalies, we draw attention to possible relationship or association between heterozygous missense variant in EYA4 gene and concurrent otosclerosis and SSCND.

The coexistence of otosclerosis and SSCND syndrome is rarely observed. Differential diagnosis of otosclerosis and SSCD syndrome is possible with clinical and audiologic findings and thin-slice temporal CT. This case report is the first to show two separate entities observed together in monozygotic twins who had a heterozygous missense variant in the EYA4 gene. To the best of our knowledge, the EYA4 gene has not yet been associated with either otosclerosis or SSCND syndrome. ES data may imply possible causal association between variants in EYA4 gene and concurrent otosclerosis and SSCD. Recent studies further support our findings as well. Exome/genome sequencing of other cases who are diagnosed with the coexistence of otosclerosis and SSCND will provide further evidence and confirmation in order to enlighten the etiology of this association.

\section{Supplementary Materials}

The online-only Data Supplement is available with this article at https://doi.org/10.7874/jao.2021.00381.

\section{Acknowledgments}

This study was supported in part by the US National Human Genome Research Institute (NHGRI) and National Heart Lung and Blood Institute (NHBLI) to the Baylor-Hopkins Center for Mendelian Genomics (BHCMG, UM1 HG006542).

\section{Conflicts of interest}

The authors have no financial conflicts of interest.

\section{Author Contributions}

Conceptualization: F Ceyda Akin Ocal. Data curation: F Ceyda Akin Ocal, Haluk Kavus. Formal analysis: F Ceyda Akin Ocal, Haluk Kavus. Funding acquisition: Davut Pehlivan. Investigation: F Ceyda Akin Ocal, Haluk Kavus. Methodology: all authors. Project administration: all authors. Visualization: F Ceyda Akin Ocal, Haluk Kavus. Writing — original draft: all authors. Writing — review \& ed- iting: Bulent Satar, Davut Pehlivan. Approval of final manuscript: all authors.

\section{ORCID iDs}

F Ceyda Akin Ocal https://orcid.org/0000-0001-7212-2208

Haluk Kavus https://orcid.org/0000-0003-3650-7498

Bulent Satar https://orcid.org/0000-0002-1079-2393

Davut Pehlivan https://orcid.org/0000-0001-5788-0270

\section{REFERENCES}

1) Lehmann M, Ebmeyer J, Upile T, Sudhoff HH. Superior canal dehiscence in a patient with three failed stapedectomy operations for otosclerosis: a case report. J Med Case Rep 2011;5:47.

2) Baxter M, McCorkle C, Trevino Guajardo C, Zuniga MG, Carter AM, Della Santina CC, et al. Clinical and physiologic predictors and postoperative outcomes of near dehiscence syndrome. Otol Neurotol 2019;40:204-12.

3) Karczewski KJ, Francioli LC, Tiao G, Cummings BB, Alföldi J, Wang $\mathrm{Q}$, et al. The mutational constraint spectrum quantified from variation in 141,456 humans. Nature 2020;581:434-43.

4) Lek M, Karczewski KJ, Minikel EV, Samocha KE, Banks E, Fennell T, et al. Analysis of protein-coding genetic variation in 60,706 humans. Nature 2016;536:285-91.

5) The Atherosclerosis Risk in Communities (ARIC) study: design and objectives. The ARIC investigators. Am J Epidemiol 1989;129: 687-702.

6) Fu W, O'Connor TD, Jun G, Kang HM, Abecasis G, Leal SM, et al. Analysis of 6,515 exomes reveals the recent origin of most human protein-coding variants. Nature 2013;493:216-20.

7) Hope A, Fagan P. Latent superior canal dehiscence syndrome unmasked by stapedotomy for otosclerosis. J Laryngol Otol 2010;124: 428-30.

8) Ungar OJ, Handzel O, Cavel O, Oron Y. Superior semicircular canal dehiscence with concomitant otosclerosis-a literature review and case discussion. Clin Case Rep 2018;6:2364-70.

9) Heidenreich KD, Kileny PR, Ahmed S, El-Kashlan HK, Melendez TL, Basura GJ, et al. Superior canal dehiscence syndrome affecting 3 families. JAMA Otolaryngol Head Neck Surg 2017;143:656-62.

10) Thys M, Van Camp G. Genetics of otosclerosis. Otol Neurotol 2009; 30:1021-32.

11) Foster MF, Backous DD. Clinical evaluation of the patient with otosclerosis. Otolaryngol Clin North Am 2018;51:319-26.

12) Richard C, Linthicum FH Jr. An unexpected third window in a case of advanced cavitating otosclerosis. Otol Neurotol 2012;33:e47-8.

13) Borsani G, DeGrandi A, Ballabio A, Bulfone A, Bernard L, Banfi S, et al. EYA4, a novel vertebrate gene related to Drosophila eyes absent. Hum Mol Genet 1999;8:11-23.

14) Depreux FF, Darrow K, Conner DA, Eavey RD, Liberman MC, Seidman CE, et al. Eya4-deficient mice are a model for heritable otitis media. J Clin Invest 2008;118:651-8.

15) Gana S, Valetto A, Toschi B, Sardelli I, Cappelli S, Peroni D, et al. Familial Interstitial 6q23.2 deletion including Eya4 associated with otofaciocervical syndrome. Front Genet 2019;10:650. 